\title{
EXPÉRIENCE ACQUISE DANS LE DIABÈTE
}

\author{
C Ernould ${ }^{1}$, MP Graff ${ }^{2}$, JP Bourguignon ${ }^{1}$ \\ ${ }^{1}$ Unité d'endocrinologie-diabétologie pédiatrique, département universitaire de pédiatrie, 45, quai G-Kurth, \\ 4020 Liège, Belgique ; \\ ${ }^{2}$ Action Jeunes diabétiques de l'Office de la naissance et de l'enfance.
}

Le survol proposé ici décrit les étapes marquantes d'une trentaine d'années d'expérience dans la prise en charge et l'accompagnement d'enfants et d'adolescents diabétiques. Les objectifs principaux ont été de rendre ces patients aussi bien équilibrés que possible au plan métabolique, mais également aussi autonomes qu'ils puissent l'être en dépit du diabète. Ces enjeux supposent l'acquisition d'aptitudes techniques et psychologiques autorisant le jeune à gérer lui-même les différents domaines du diabète. L'éducation destinée à rendre ces jeunes (et leurs parents) compétents, a été renforcée durant 17 années par le concours d'infirmières spécialisées en diabétologie pédiatrique. Leur double mission sur le terrain (domicile, école, mouvements de jeunesse, clubs sportifs, etc) a été, d'une part de proposer aux familles la mise en route concrète d'une pédagogie réaliste, d'autre part d'instruire le diabétologue-pédiatre des domaines du possible, de l'incertain, de l'inaccessible, pour chacun des patients. Ce retour d'informations a probablement constitué pour l'équipe soignante, jaugeant la "faisabilité », l'incitant majeur à s'interroger sur l'obligation de moyens pour s'imposer, jusqu'à un certain point, une obligation de résultats.

\section{6}

C'est l'année où l'un de nous se voit confier la prise en charge des jeunes diabétiques. Il découvre :

- que ces patients sont atteints d'une maladie grave, définitive, contraignante, au décours fluctuant et au pronostic à long terme réservé ;

- que les enfants, les adolescents, leurs parents sont soumis à des prescriptions (analyses, injections, régime fixe à l'époque) ;

- qu'ils doivent s'y tenir et qu'ils n'ont guère le droit d'initiative ;

- qu'ils rendent compte de l'évolution à la consultation toutes les 6 semaines apportant un carnet servilement documenté et des urines pour glucosurie de 24 heures et se voyant prélever une glycémie, ces deux dernières informations pesant lourdement dans le " jugement » du médecin ; 
- que les réhospitalisations sont régulièrement programmées pour certains patients ;

- et que le système n'empêche pas les réhospitalisations en urgence, essentiellement pour (acido)-cétose.

À l'époque, $26 \%$ des patients sont réhospitalisés, en moyenne 36 jours/an (tableau I).

Tableau I. Évolution de l'hospitalisation au début du diabète et par la suite d'enfants et adolescents diabétiques suivis au département de pédiatrie de l'université de Liège de 1966 à 1994.

\begin{tabular}{|c|c|c|c|c|c|}
\hline Année & 1966 & 1976 & 1981 & 1992 & 1994 \\
\hline Nombre de patients suivis & 27 & 52 & 81 & 86 & 114 \\
\hline Hospitalisation au début du diabète & $6(22.2 \%)$ & $8(15,4 \%)$ & $1(1.2 \%)$ & 8 (9,3 \%) & $11(9,6 \%)$ \\
\hline Nombre annuel de patients & 98 & 68 & 5 & 89 & 97 \\
\hline Durée moyenne de séjour (en jours) & 16,3 & 8,5 & 5 & 11,1 & 8,3 \\
\hline \multicolumn{6}{|l|}{ Réhospitalisations } \\
\hline Nombre annuel de patients & $7(25,9 \%)$ & $7(13.5 \%)$ & $7(8,6 \%)$ & $6(7 \%)$ & $17(14,9 \%)$ \\
\hline Nombre de journées/an & 250 & 45 & 61 & 41 & 89 \\
\hline Durée moyenne de séjour (en jours) & 35,7 & 6,4 & 8,7 & 6,8 & 5,2 \\
\hline & & \multicolumn{4}{|c|}{$\begin{array}{c}\text { Action « Jeunes tabétiques » } \\
\text { ONE } \\
1977-1993\end{array}$} \\
\hline
\end{tabular}

\section{6-1976}

La réaction devant la subordination au paternalisme, sinon au pouvoir médical, et l'échec fréquent qui l'accompagne se concrétisent par la mise en place progressive d'une «éducation » du patient et de ses proches:

- à l'occasion des hospitalisations au début du diabète, dont cependant la durée moyenne de séjour passe de 16,3 à 8,5 jours ;

- à partir de 1969 en encourageant les jeunes à participer aux camps de vacances et en y étant nous-mêmes partie prenante depuis 1970 et pendant plus de 20 ans ;

- en élargissant le champ des échanges à la consultation en dépit d'une durée (30-40 minutes, parfois plus) encore insuffisante. Cet ensemble de moyens s'assortit d'une réduction des réhospitalisations : en 1976, $13 \%$ des patients sont réhospitalisés (réduction de $50 \%$ en 10 ans) pour une durée moyenne de séjour de 6,4 jours/an (réduction de $82 \%$ en 10 ans).

Convaincu qu'une juste appréciation du quotidien impose d'aller voir sur place, constatant que le médecin de famille n'est pas partie prenante de cette évaluation, une première estimation des actes faits par le patient est réalisée en 1972-73 par une infirmière spécialisée dont le passage à domicile concourt à l'augmentation du nombre de patients rendus, à nouveau, capables de faire 
correctement les actes techniques qui leur avaient été confiés [1]. Fort de cette expérience, nous sensibilisons un membre de la famille royale venu rendre visite au camp de 1976. Sa conclusion sera la première étape des démarches que nous accomplirons pendant 1 année auprès de l'Office de la naissance et de l'enfance (ONE) pour que cette institution crée l'action « Jeunes diabétiques » (JD) en engageant des infirmières spécialisées en diabétologie pédiatrique dont la mission sera de prolonger sur le terrain, en la concrétisant, l'action éducative entreprise en hospitalisation et en consultation.

\section{7-1993}

C'est le temps de l'action JD de l'ONE qui démarre petitement avec deux infirmières pour l'ensemble de la Belgique pour tourner à plein régime à partir de $1983 \mathrm{avec}$, en communauté française, quatre équivalents temps plein pour 360 patients enregistrés. Cette action sera réduite en communauté française à $50 \%$ de ses effectifs infirmiers en septembre 1992 et sera clôturée au 31 décembre 1993, la communauté française étant chargée du volet préventif, la charge curative des soins de santé (prévention tertiaire comprise) devant rester ou revenir au ministère fédéral pour l'ensemble du pays. Les démarches nombreuses auprès de ce ministère n'ont pas abouti à ce jour ; les informations récentes (décembre 1995) nous permettent d'espérer un financement de l'action des infirmières par le ministère fédéral compétent.

Cette action des infirmières, sur le terrain, allait nous faire mieux comprendre, pour mieux les appréhender, les «falsifications » de carnet que les premiers contrôles glycémiques à domicile avaient mis en évidence dans $50 \%$ des cas [2] et que les dosages de l'hémoglobine glycosylée devaient confirmer [3]. Ce phénomène n'est en fait que la partie émergée de l'iceberg, un révélateur de la persistance d'une pression médicale et parentale tout autant que d'une écoute insuffisante à l'égard du patient [4]. D'une manière plus générale, ces infirmières ont mis en lumière le vécu des patients et nous ont ainsi permis de préciser davantage nos objectifs pédagogiques et les modalités d'éducation [5]. Durant cette fructueuse période, les réhospitalisations ont continué de décroître pour ne plus concerner que $7 \%$ des patients. Outre les avantages évidents pour les jeunes diabétiques de rester intégrés dans leurs milieux de vie, l'économie pour les budgets de santé est importante, ce que nous ne manquons pas de rappeler à nos interlocuteurs des mondes politique et administratif.

\section{Après 1993}

L'actualité est faite d'amertume et d'espoir. Nos regrets de l'absence des infirmières sont sans doute faibles à côté de la déception régulièrement témoignée par les familles et la détresse que connaissent les parents dans les premiers mois du diabète de leur enfant. Notre permanence téléphonique quotidienne, mise en place depuis une dizaine d'années ( 15 min le matin, 45 min en soirée), ne compense pas ce que nous considérons comme une carence grave des pouvoirs 
publics. À l'école, la lacune est tout aussi perceptible (l'information scolaire occupait les infirmières 2 mois/an) et ce ne sont pas les séances d'informations que nous proposons une fois par an aux enseignants qui apportent une solution satisfaisante au problème. Enfin, la réhospitalisation s'accroît et a concerné 15\% des patients en 1994.

L'espoir, c'est l'écoute récente des gens du pouvoir fédéral sensibilisés peut-être par ce dernier point et ses retombées économiques. Une décision positive servirait assurément les patients dont l'un des enjeux, le principal dans l'esprit des médecins sans doute, est la meilleure santé possible loin dans le XXIe siècle. 


\section{Références}

1 Ernould C. Efficacité d'une infirmière se rendant à domicile de jeunes diabétiques. Rev Méd Liège 1973: 28: 199-203

2 Ernould C. Graff MP. Interest of blood glucose value determined at home in juvenile diabtes. Acta Paediatr Belg 1978: 31: 199-203

3 Ernould C. Bourguignon JP. Individual significances of $\mathrm{Hb}$ Alc diabetic children and adolescents according to apparent and actual degree of control. Bull ISGD 1980; 4: 27-8

4 Ernould C. Éducation. In : Czemichow P, Dorchv H. eds. Diabétologie pédiatrique. Paris : Dcin. 1989: $397-426$

5 Ernould C. Diabète insulino-dépendant de l'enfant. In : Assal JP. Ekoe JM, Lefèbvre Slama PG, eds. Reconnaître, comprendre, traiter : le diabète sucré. Paris : Maloine, Edisem, St Hyacinte Québec, 1994: 27-43 